\title{
The Equivalence of Certain Norms on the Heisenberg Group
}

\author{
Murphy E. Egwe \\ Department of Mathematics, Universty of Ibadan, Ibadan, Nigeria \\ Email: murphy.egwe@ui.edu.ng,me_egwe@yahoo.co.uk
}

Received April 25, 2013; revised May 29, 2013, accepted June 23, 2013

Copyright (C) 2013 M. E. Egwe. This is an open access article distributed under the Creative Commons Attribution License, which permits unrestricted use, distribution, and reproduction in any medium, provided the original work is properly cited.

\section{ABSTRACT}

Let $I H_{n}$ be the $(2 n+1)$-dimensional Heisenberg group. In this paper, we shall give among other things, the properties of some homogeneous norms relative to dilations on the $I H_{n}$ and prove the equivalence of these norms.

Keywords: Heisenberg Group; Heisenberg Norms; Equivalent Norms; Homogeneous Group

\section{Introduction}

The Heisenberg group (of order $n$ ), $I H_{n}$ is a noncommutative nilpotent Lie group whose underlying manifold is $\mathbb{C}^{n} \times I R$ with coordinates $(z, t)=\left(z_{1}, z_{2}, \cdots, z_{n}, t\right)$ and group law given by

$$
\begin{aligned}
& (z, t)\left(z^{\prime}, t^{\prime}\right)=\left(z+z^{\prime}, t+t^{\prime}+2 \Im m z \cdot z^{\prime}\right) \\
& \text { where } z \cdot z^{\prime}=\sum_{j=1}^{n} z_{j} \bar{z}_{j} \quad z \in \mathbb{C}^{n}, t \in I R .
\end{aligned}
$$

Setting $z_{j}=x_{j}+y_{j}$, then $\left(x_{1}, x_{2}, \cdots, x_{n}, y_{1}, y_{2}, \cdots, y_{n}, t\right)$ forms a real coordinate system for $I H_{n}$. In this coordinate system, we define the following vector fields:

$$
X_{j}=\frac{\partial}{\partial x_{j}}+2 y_{j} \frac{\partial}{\partial t}, Y_{j}=\frac{\partial}{\partial y_{j}}-2 x_{j} \frac{\partial}{\partial t}, T=\frac{\partial}{\partial t} .
$$

The set $\left\{X_{1}, X_{2}, \cdots, X_{n}, Y_{1}, Y_{2}, \cdots, Y_{n}, T\right\}$ forms basis for the left invariant vector fields on $I H_{n}$ [1]. These vector fields span the Lie algebra $\mathfrak{h}_{n}$ of $I H_{n}$ and the following commutation relations hold:

$$
\left[Y_{j}, X_{k}\right]=4 \delta_{j k} T,\left[Y_{j}, Y_{j}\right]=\left[X_{j}, T\right]=\left[Y_{j}, T\right]=0 .
$$

Similarly, we obtain the complex vector fields by setting

$$
\left.\begin{array}{l}
Z_{j}=\frac{1}{2}\left(X_{j}-i Y_{j}\right)=\frac{\partial}{\partial z_{j}}+i \bar{z} \frac{\partial}{\partial t} \\
\bar{Z}_{j}=\frac{1}{2}\left(X_{j}+Y_{j}\right)=\frac{\partial}{\partial \bar{z}_{j}}-i z \frac{\partial}{\partial t}
\end{array}\right\}
$$

In the complex coordinate, we also have the commutation relations

$$
\begin{aligned}
& {\left[Z_{j}, \bar{Z}_{k}\right]=-2 \delta_{j k} T,} \\
& {\left[Z_{j}, Z_{k}\right]=\left[\bar{Z}_{j}, \bar{Z}_{k}\right]=\left[Z_{j}, T\right]=\left[\bar{Z}_{j}, T\right]=0 .}
\end{aligned}
$$

If we identify $I H_{n}$ with $I R^{2 n+1}$, then each element of $I H_{n}$ is given by $u=(x, y, t) \in I R^{n} \times I R^{n} \times I R$ and the group law becomes

$$
(x, y, t)\left(x^{\prime}, y^{\prime}, t^{\prime}\right)=\left(x+x^{\prime}, y+y^{\prime}, t+t^{\prime}+2\left\langle x, y^{\prime}\right\rangle\right)
$$

where $(x, y) \mapsto\left\langle x, y^{\prime}\right\rangle=\sum_{j=1}^{n} x_{j} y_{j}$ denotes the scalar product of $I R^{n}$. The neutral element $1_{I H_{n}}$ of $I H_{n}$ is of the form $(0,0,0)$ and the inverse element

$$
(x, y, t)^{-1}=(-x,-y,-t+\langle x, y\rangle) \text {. }
$$

The centre of $I H_{n}$ is given by

$$
\mathcal{Z}=\{(0,0, t): t \in I R\}
$$

and therefore isomorphic to the additive locally compact topological group IR. The Haar measure on $I H_{n}$ is the Lebesgue measure $\mathrm{d} x \mathrm{~d} y \mathrm{~d} t$ on $I^{2 n} \times I R$ [1].

On the group, we introduce the group $\left\{\delta_{r}: 0<r<\infty\right\}$ of dilations defined for each element $u=(z, t)$ of $I H_{n}$ by $\delta_{r}(z, t)=\left(r z, r^{2} t\right)$ on the complex coordinates and by $\delta_{r}(x, y, t)=\left(r x, r y, r^{2} t\right)$ on the real coordinates. The family of dilations $\left\{\delta_{\lambda}\right\}_{\lambda}>0$ forms a one-parameter group of automorphisms of $I H_{n}$ Indeed, we have the following properties of this family of dilations. 
(i) $\delta_{r s}(u)=\delta_{r}\left(\delta_{s}(u)\right), \forall u \in I H_{n}, r, s>0$,

(ii) $\delta_{r}\left(u \cdot u^{\prime}\right)=\delta_{r}(u) \delta_{r}\left(u^{\prime}\right)$. Moreover,

(iii) $\left(\delta_{r}\right)^{-1}(u)=\delta_{r-1}(u)$. Properties (i) and (iii) can be easily seen $[2,3]$. To see (ii), we notice that: For $(x, y, t),\left(x^{\prime}, y^{\prime}, t^{\prime}\right) \in I H_{n}$ and $\delta_{r}: I H_{n} \rightarrow I H_{n}$, we have

$$
\begin{aligned}
& \delta_{r}\left((x, y, t)\left(x^{\prime}, y^{\prime}, t^{\prime}\right)\right) \\
= & \delta_{r}\left(x+x^{\prime}, y+y^{\prime}, t+t^{\prime}+2\left(x y^{\prime}-x^{\prime} y\right)\right) \\
= & \left(r x+r x^{\prime}, r y+r y^{\prime}, r^{2} t+r^{2} t^{\prime}+2 r^{2}\left(x y^{\prime}-x^{\prime} y\right)\right) \\
= & \delta_{r}(x, y, t) \delta_{r}\left(x^{\prime}, y^{\prime}, t^{\prime}\right) .
\end{aligned}
$$

With these dilations as automorphisms of $\left(I R^{2 n} \times I R, \circ\right)$, $I H_{n}:=\left(I R^{2 n} \times I R, \circ, \delta_{r}\right)$ becomes a stratified Lie group whose generators are the defined vector fields [4]. Similarly, $I H_{n}$ and its Lie structure equipped with this family of dilations is a homogeneous group of dimension $(2 n+1)$ [5].

\section{Homogeneous Norms on $\mathbf{I H}$}

Definition 2.1: A norm on the Heisenberg group, is a function

$$
||_{I H_{n}}: I H_{n} \rightarrow[0, \infty)
$$

satisfying the following properties:

(i) $\left|\delta_{r} u\right|_{I H_{n}}=r|u|_{I H_{n}}$,

(ii) $|u|=0 \Leftrightarrow u=0$,

(iii) $\left|u^{-1}\right|=|u|$,

(iv) $\left|u_{1} u_{2}\right| \leq\left|u_{1}\right|+\left|u_{2}\right|$ for all $u$ and $r>0$, where $u=(z, t)$.

The value $|(z, t)|_{I H_{n}}=\left(|z|^{4}+t^{2}\right)^{1 / 4}$ is called the Heisenberg distance of $(z, t)$ from the origin and $\left(|z|^{4}+t^{2}\right)^{1 / 4}<1$ is the Heisenberg unit ball [6]. We say the norm in (2.1) is homogeneous of degree $Q$ with respect to the dilations if for any $u \in I H_{n}$, we have $\left|\delta_{r} u\right|_{I H_{n}}=r^{Q}|u|_{I H_{n}}$. The value given by

$$
|(z, t)|:=\left(|z|^{4}+16 t^{2}\right)^{1 / 4}=\left.|| z\right|^{2} \pm\left. 4 i t\right|^{1 / 2}
$$

is the popular Koranyi norm on $I H_{n}$ which is always positive definite [7].

Property (i) is the homogeneity of the Heisenberg norm while property (iv) indicates the subadditivity of the Heisenberg norm. The proof of properties (i)-(iii) is trivial and that of (iv) can be found in [8].

Following [9], we shall further define the following norms on $I H_{n}$. For $u=(z, t)$, define

$$
\left.\begin{array}{l}
|u|_{0}=\max \left\{\left|z_{1}\right|, \cdots,\left|z_{n}\right|,|t|^{1 / 2}\right\} \\
|u|_{1}=1 \Leftrightarrow|z|^{2}+t^{2}=1 \text { and extended by homogeneity. } \\
|u|_{2}=\left(\left|z_{1}\right|^{4}+\cdots+\left|z_{n}\right|^{4}+t^{2}\right)^{1 / 4}=\left(\sum_{j=1}^{n}\left|z_{j}\right|^{4}+|t|^{2}\right)^{1 / 4}
\end{array}\right\}
$$

We notice that $|u|_{0}$ gives a choice which is not smooth away from the origin. The norm $|u|_{2}=\left(|z|^{4}+t^{2}\right)^{1 / 4}$ and the properties above do not uniquely determine the norm. For if $\phi$ is positive, smooth away from 0 , and homogeneous of degree 0 in the Heisenberg group dilation structure, then $|u|_{h}^{*} \equiv \phi(u)|u|_{h}$ gives another norm [10].

Since $I H_{n}=\mathbb{C}^{n} \times I R$, it can be equipped with the Euclidean norm in $I R^{2 n+1}$ denoted by $|u|_{e}$ and defined by

$$
|u|_{e}=\left(|z|^{2}+|t|^{2}\right)^{1 / 2}, u=(z, t) \in I H_{n} .
$$

We have the following:

Proposition 2.3 [10]: For $|u|_{e}^{2} \leq \frac{1}{2}$, we have

$$
|u|_{e} \leq|u|_{I H_{n}} \leq|u|_{e}^{1 / 2} \text {. }
$$

We notice however, that this norm is not homogeneous. In what follows, we show that homogeneous norms on the Heisenberg group are equivalent following [10].

Lemma 2.4: Let ||$_{I H_{n}}$ be a homogeneous norm on $I H_{n}$ Then, there is a constant $M>0$ such that

$$
M^{-1}|u|_{2} \leq|\cdot|_{I H_{n}} \leq M|u|_{2} \forall u \in I H_{n}
$$

where $|u|_{2}$ is as defined in (2.2).

Proof: Now observe that $|u|_{I H_{n}}$ is homogeneous of degree $2 n+2$ and by hypothesis, $|\cdot|_{2}$ is homogeneous. Let

$$
\begin{aligned}
& R:=\sup \left\{|u|_{I H_{n}}:|u|=1\right\}<\infty \\
& \text { and } r:=\inf \left\{|u|_{I H_{n}}:|u|=1\right\}>0
\end{aligned}
$$

and set

$$
M:=\max \left\{R, \frac{1}{r}\right\} .
$$

Now, if we identify $I H_{n}$ as $I R^{2 n+1}$, then sup is actually a maximum and inf is a minimum. Thus $M \neq 0$ exists and the inequality in the theorem holds. This is possible since $R<\infty$ and $r>0$ follows from the fact that $\{u:|u|=1\}$ is a compact subset of $I H_{n}$ not containing the origin and $|\cdot|_{I H_{n}}$ is a continuous function 
which is strictly positive in $I H_{n} \backslash\{0\}$.

Corollary 2.5: For every fixed homogeneous norm ||$_{I H_{n}}$ on $I H_{n}$ there exists a constant $M>0$ such that

$$
M^{-1}|u|_{I H_{n}} \leq\left|u^{-1}\right|_{I H_{n}} \leq M|u|_{I H_{n}} \quad \forall x \in I H_{n} .
$$

Proof: We notice that the norm function is continuous and therefore, $|x|=\left|x^{-1}\right|$. Now consider the the group of dilations $\left\{\delta_{r}: r>0\right\}$ on $I H_{n}$ Then $\delta_{r}\left(x^{-1}\right)=\left(\delta_{r}(x)\right)^{-1}$ is an automorphism of $G$. Therefore, by Lemma 2.4, the result follows.

Theory 2.6: Any two homogeneous norms on $I H_{n}$ are equivalent.

Proof: We apply the previous method as follows: Let

$$
W:=\left\{u \in I H_{n}:|u|^{\delta_{r}} \leq 1\right\}
$$

and define $\varphi: W \rightarrow[0, \infty)$ by

$$
\varphi(u)=|u|^{\delta_{r}}=r^{q}|u|, \quad q \geq 1 .
$$

Then

$$
p:\left(I H_{n},|u|_{1}\right) \rightarrow\left(I H_{n},|u|_{2}\right)
$$

is obviously continuous by the homogeneity property with respect to $|u|_{1}$. Since $W$ is bounded with respect to $|u|_{1}, \varphi$ attains it bounds and therefore, $\sup \varphi$ exists. Thus, $\exists M>0$ such that $\varphi(u) \leq M$. If $0 \neq u \in I H_{n}$, then there exists $r, R \geq 0$ such that $\delta_{R}(u)|u|_{2}^{\delta_{r}} \in I H_{n}$ so that

$$
\varphi\left(\frac{\delta_{R}(u)}{|u|_{2}^{\delta_{r}}}\right) \leq \frac{R|u|}{r|u|}=R \frac{1}{r} \leq K M=M^{\prime} .
$$

The theorem then follows by Lemma 2.4.

\section{REFERENCES}

[1] R. Howe, "On the Role of the Heisenberg Group in Har- monic Analysis," Bulletin of the American Mathematical Society, Vol. 3, 1980, pp. 821-843.

doi:10.1090/S0273-0979-1980-14825-9

[2] D. Muller, “Analysis of Invariant PDO's on the Heisenberg Group," ICMS-Instructional Conference, Edinburg, 6-16 April 1999, pp. 1-23.

[3] M. E. Egwe, "Aspects of Harmonic Analysis on the Heisenberg Group," Ph.D. Thesis, University of Ibadan, Ibadan, 2010.

[4] A. Bonfiglioli, E. Lanconelli and F. Uguzzoni, "Stratified Lie Groups and Potential Theory for Their Sub-Laolacians (Springer Monographs in Mathematics)," Springer, Berlin, 2007.

[5] G. B. Folland and E. M. Stein, "Estimate for the Complex and Analysis on the Heisenberg Group," Communications on Pure and Applied Mathematics, Vol. 27, No. 4, 1974, pp. 429-522. doi:10.1002/cpa.3160270403

[6] G. B. Folland, "A Fundamental Solution for a Subelliptic Operator," Bulletin of the American Mathematical Society, Vol. 79, No. 2, 1973, p. 373. doi:10.1090/S0002-9904-1973-13171-4

[7] J. R. Lee and A. Naor, " $L_{p}$ Metrics on the Heisenberg Group and the Geomans-Linial Conjecture," Proceedings of the 47th Annual IEEE Symposium on Foundations of Computer Science, Berkeley, 21-24 October 2006, pp. 99-108.

[8] J. Cygan, "Subadditivity of Homogeneous Norms on Certain Nilpotent Lie Groups," Proceedings of the American Mathematical Society, Vol. 83, 1981, pp. 69-70. doi:10.1090/S0002-9939-1981-0619983-8

[9] N. Laghi and N. Lyall, "Strongly Singular Integral Operators Associated to Different Quasi-Norms on the Heisenberg Group," Mathematical Research Letters, Vol. 14, No. 5, 2007, pp. 825-238.

[10] S. G. Krantz, "Explorations in Harmonic Analysis with Applications to Complex Function Theory and the Heisenberg Group," Birkhauser, Boston, 2009. 